ISSN 1997-5902

\title{
Bio-indicateurs de la fertilité des sols et la perception paysanne à l'Ouest du Niger
}

\author{
MOUSSA MAMOUDOU Boubacar1*, RABIOU Habou², INOUSSA Maman Maârouhi ${ }^{1}$, BAKASSO \\ Yacoubou 1, MAHAMANE Ali 1,3 \\ 1. Laboratoire Garba Mounkaila, Département de Biologie, Faculté des Sciences et Techniques, Université Abdou \\ Moumouni de Niamey, Niger. \\ 2. Université de Maradi, Département de biologie, Faculté des Sciences et techniques, Niger \\ 3. Université de Diffa, Niger \\ *Auteur correspondant : Moussa Mamoudou Boubacar boubacar_mac@yahoo.fr, 0022797125559
}

Original submitted in on $4^{\text {th }}$ March 2015. Published online at www.m.elewa.org on 30th May 2015 http://dx.doi.org/10.4314/jab.v89i1.3

\section{RÉSUMÉ :}

Objectif : Cette étude réalisée à l'Ouest du Niger dans les Communes de Simiri et de Tamou a pour objectif d'identifier des bio-indicateurs des sols fertiles et des sols dégradés selon la perception paysanne et des paramètres environnementaux.

Méthodologie : des enquêtes auprès des populations locales et des relevés sur la strate herbacée ont été réalisés.

Résultats : Les résultats montrent qu'un sol est considéré fertile par les paysans si le rendement en grains de mil est supérieur à $800 \mathrm{~kg} / \mathrm{ha}$ à Tamou et à $400 \mathrm{~kg} / \mathrm{ha}$ à Simiri. De plus, les espèces indicatrices de la fertilité des sols sont: Cenchrus biflorus (Simiri), Pennisetum pedicellatum et Diheteropogon hagerupii (Tamou). Cependant, Mitracarpus scaber (Simiri) et Sida cordifolia (Tamou) sont indicatrices des sols dégradés.

Conclusion et applicabilité des résultats : Cette étude réalisée à l'Ouest du Niger a permis d'identifier des bioindicateurs de la dégradation des sols. Ces résultats permettront aux paysans d'apprécier le niveau de fertilité des champs nécessaire pour leur mise en jachère ou leur remise en culture. Ils constituent aussi un outil d'aide à la décision pour les décideurs dans la lutte contre la dégradation des terres.

Mots clé : Sol ; fertilité ; dégradation ; indicateurs paysans ; Niger

\section{Bio-indicators of soil fertility and farmers' perceptions in western Niger}

Abstract:

Objective: This study was conducted in western Niger in the Municipalities of Simiri and Tamou. It aims to identify biological indicators of fertile or degraded soil by farmers' perceptions and environmental parameters. Methodology: surveys of the local population and inventory of the herbaceous cover has was done. Result: The results showed that soil is considered fertile if the yield of millet grains is greater than $800 \mathrm{~kg} / \mathrm{ha}$ at Tamou and $400 \mathrm{~kg} / \mathrm{ha}$ at Simiri. In addition, presences of Cenchrus biflorus at (Simiri) and Pennisetum pedicellatum and Diheteropogon hagerupii at (Tamou) are indicators of soil fertility while Mitracarpus scaber at (Simiri) and Sida cordifolia at (Tamou) is synonymous to soil degradation.

Conclusion and applicability of the results: In this study, the bio-indicators of land degradation was identifed in western Niger. These results will enable farmers to assess the level of fertility of the fields for the fallow or 
brought back into cultivation. They are also a support tool for decision makers in the fight against land degradation.

Key words: soil; fertility; degradation; local people bio-indicators; Niger

\section{INTRODUCTION}

La production agricole constitue la principale source d'activité économique en Afrique Subsaharienne (FAO, 2003). Cependant la dégradation des agrosystèmes constitue une contrainte majeur pour cette production dans la zone semi-aride de l'Afrique de l'Ouest (Pontanier et al., 1995). Les facteurs majeurs de cette dégradation sont : la faible fertilité intrinsèque des sols et les pluies peu abondantes et intense désagrégeant les sols en surface en les rendant sensibles à l'érosion (Bationo et Buerkert, 2001; Casenave et Valentin, 1989). En conséquence, les rendements sont relativement faibles et affectent négativement la sécurité alimentaire des populations au Sahel en général et au Niger en particulier. A l'Ouest du Niger, la faible fertilité potentiel de ces sols, combinée aux péjorations climatiques se traduit par une diminution de la productivité des terres. En effet, pour évaluer ces différents changements d'état ou ces modifications de fonctionnement du milieu rural,

\section{MATERIEL ET METHODES}

Zone d'étude : Cette étude a été menée dans deux Communes rurales situées dans la partie Ouest du Niger : la Commune rurale de Simiri au Nord, entre $13^{\circ} 50^{\prime}$ et $14^{\circ} 17^{\prime}$ de latitude Nord, et $01^{\circ} 50^{\prime}$ et $02^{\circ} 40^{\prime}$ de longitude Est ; et la Commune rurale de Tamou au Sud, entre $12^{\circ} 24^{\prime}$ et $13^{\circ} 30^{\prime}$ de latitude Nord, et $01^{\circ} 40^{\prime}$ et induits par les perturbations naturelles et par les activités humaines, l'observateur fait recourt à des indicateurs (Roger P. et Christian F., 2002). Un indicateur est un paramètre qui fournit des informations sur un phénomène qui affecte un système ou sur l'état des composants du système (Loireau., 1998). C'est ainsi que les paysans, en raison des connaissances longuement acquises de leurs milieux se basent sur des indicateurs pour évaluer la qualité de leurs terres (M'Biandoun et Bassala, 2007). Cette étude se propose d'identifier des bio-indicateurs de la fertilité des sols selon la perception paysanne et des paramètres environnementaux. II s'agit spécifiquement (a) d'inventorier les principales cultures et techniques culturales ; (b) d'identifier les espèces indicatrices des sols fertiles et des sols dégradés selon les perceptions des paysans et (c) d'apprécier les valeurs indicatrices de ces bio-indicateurs.

$02^{\circ} 40^{\prime}$ de longitude Est. Ces deux Communes diffèrent selon le type de climat : semi-aride de type sahélien pour Simiri et sahélo-soudanien pour Tamou (Figure 1). Les moyennes pluviométriques (1981-2002) sont de 330,8 $\mathrm{mm}$ à Simiri contre 601,7 mm à Tamou (Mahamane et al., 2013). 


\section{Moussa et al. . J. Appl. Biosci. Bio-indicateurs de la fertilité des sols et la perception paysanne à l'Ouest du Niger}

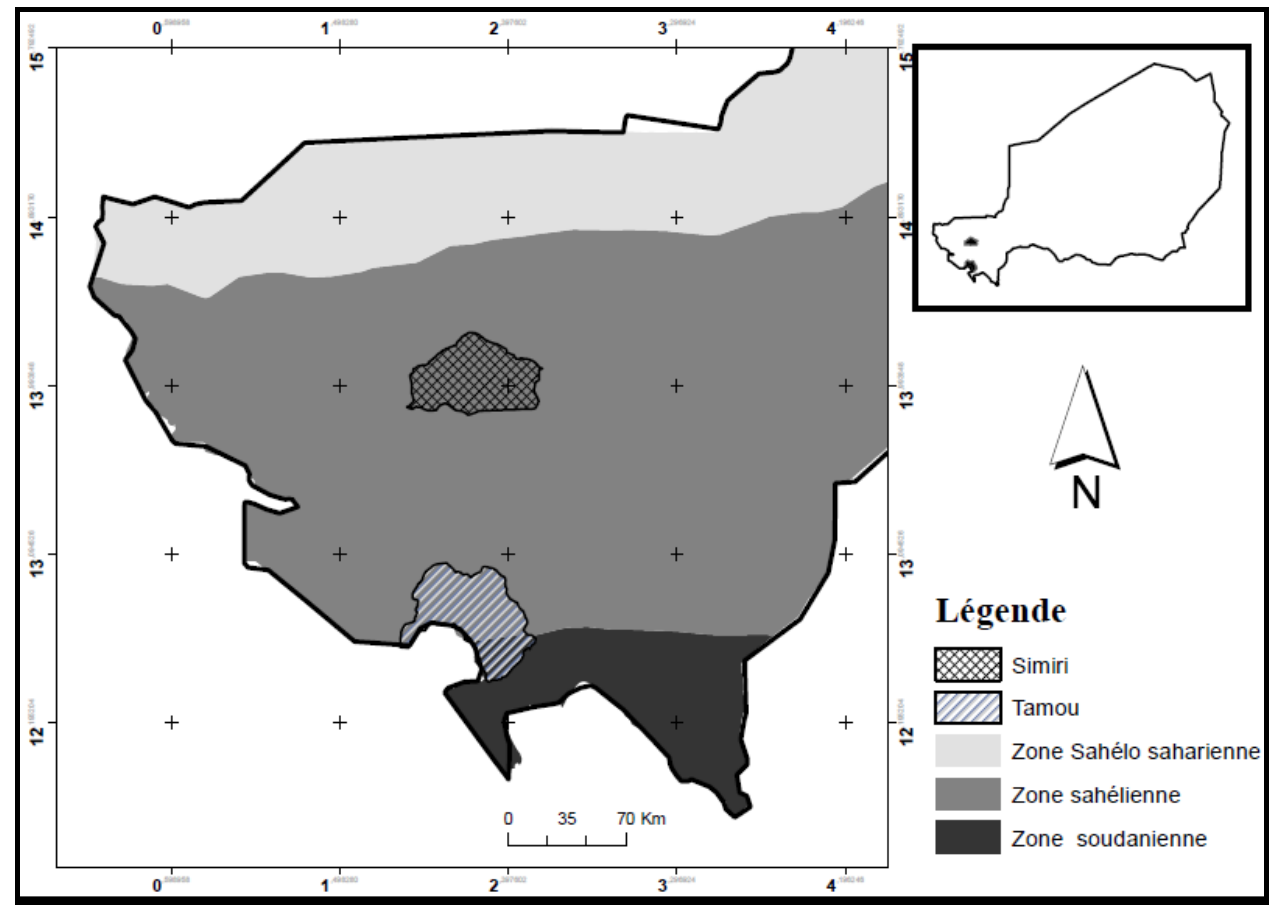

Figure 1 : Localisation des Communes de Simiri, de Tamou et les zones climatiques au Niger

La toposéquence représentative de chacune des deux Communes se caractérise par une succession de trois grands ensembles typiques des zones de plateaux ferrugineux tropicaux du Sahel, à savoir : le plateau cuirassé, le glacis et les bas-fonds (Courault et al., 1990). Des sols squelettiques de types régosols et lithosols formés sur grès argileux du continental terminal s'observent sur les plateaux (Ambouta., 1997). Le glacis est constitué de sols sableux de type ferrugineux tropicaux peu lessivés tandis que les bas-fonds et les cordons ripicoles sont caractérisés par des sols alluviaux. La végétation naturelle est constituée de steppe et de savane arbustive sur les glacis et de savane arbustive à arborée dans les bas-fonds et les formations ripicoles. Sur les plateaux, une formation contractée couramment appelée brousse tigrée ou tachetée est observée (Diouf et al., 2010). Les principales espèces ligneuses rencontrées sont Guiera senegalensis J.F.Gmel, Combretum micranthum G.Don et Combretum glutinosum Perr.ex DC. La strate herbacée est dominée par
Mitracarpus scaber Zucc., Eragrostis tremula Stend. et Cenchrus biflorus Roxb (Saadou, 1990). Les principales activités des populations sont l'agriculture et l'élevage. L'agriculture, pratiquée sur les glacis et dans les basfonds. L'élevage du gros bétail et des petits ruminants constitue la seconde activité qui génère des revenus aux populations locales.

Méthodes : La méthodologie a consisté à l'application de deux approches complémentaires. La première a été la réalisation d'enquêtes auprès des populations locales pour identifier les bio-indicateurs des sols fertiles et des sols dégradés. Ainsi, 40 paysans ont été choisis au hasard sur la liste des paysans dans chaque Commune. Les agriculteurs et les éleveurs âgés de plus de 50 ans ont été les personnes ciblées par l'enquête. Les questions ont portés sur les cultures pratiquées, les associations culturales, le rendement en grain de la culture principale $(R)$, les espèces végétales et les états de surface indicateurs des sols fertiles et des sols dégradés (Tableau 1). 

l'Ouest du Niger

Tableau $1:$ Variables et modalités utilisées

\begin{tabular}{ll}
\hline \multicolumn{1}{c}{ Variables } & \multicolumn{1}{c}{ Modalités } \\
\hline & $\mathrm{R}<400$ \\
Rendement en grain (Kg/ha) & $400<\mathrm{R}<800$ \\
& $\mathrm{R}>800$ \\
États de surface & État de surface ruisselant (ESR) \\
& État de surface infiltrant (ESI) \\
Espèce indicatrice d'un sol fertile & Présence \\
& Absence \\
Espèce indicatrice d'un sol dégradé & Présence \\
\hline
\end{tabular}

La seconde approche a consisté à la réalisation des relevés phytosociologiques de la végétation herbacée sur des sols identifiées par les paysans comme fertiles ou dégradés (Braun-Blanquet, 1932). C'est ainsi que des placettes de $100 \mathrm{~m}^{2}(10 \mathrm{~m} \times 10 \mathrm{~m})$ ont été installées sur chaque type de sol identifié par les paysans comme fertile ou dégradé. Ensuite, un code d'abondancedominance correspondant à un taux de recouvrement moyen, a été attribué aux espèces identifiées dans chaque placette (Tableau 2). Au total 80 placettes ont été prospectées soit 40 par Commune et par type de sol.

Tableau 2 : Échelle de notation

\begin{tabular}{cc}
\hline Code & Taux de recouvrement moyen (\%) \\
\hline+ & 0,5 \\
1 & 3 \\
2 & 15 \\
3 & 37,5 \\
4 & 62,5 \\
5 & 87,5 \\
\hline
\end{tabular}

\section{Traitement des données}

Identification des bio-indicateurs paysans: Une analyse factorielle de correspondance (AFC) a été réalisée sur une matrice de 29 variables (espèces indicatrices, états de surface et rendements en grain) et quatre facteurs (types de sol par Commune) pour identifier les bio-indicateurs paysans. Seuls les variables et les facteurs ayant des valeurs de contributions et de cosinus carré les plus élevés ont été retenus pour l'interprétation des axes canoniques. Le logiciel $R$ a servi à la réalisation de cette analyse ( $R$ development Core $T$., 2010).

Calcul des valeurs indicatrices des espèces: Sur la base des relevés phytosociologiques, les valeurs indicatrices des espèces ont été calculées par Commune pour chaque type de sol (fertile et dégradé). Cette valeur est obtenue par multiplication des fréquences relatives et des abondances relatives de chaque espèce par type de sol (Dufrene et Legendre, 1997). La valeur indicatrice varie de 0 (pas d'indication) à $100 \%$ (indication parfaite). Enfin, les trois espèces ayant les valeurs indicatrices les plus élevées ont été retenues. La formule suivante a permis de calculer la valeur indicatrice de chacune des espèces :

$$
V I=A r \times F r
$$

avec

$A r=\frac{A i j}{A i}$

et

$$
F r=\frac{N P U j}{N P j}
$$

Ar: Abondance relative; Fr: fréquence relative; Aij : abondance moyenne de l'espèce i dans le type de sol $\mathrm{j}$; $\mathrm{Ai}$ : total des abondances moyennes de l'espèce i dans tous les sols ; NPij : nombre de placettes du type de sol j où l'espèce $\mathrm{i}$ est présente et NPj: nombre total de placettes du type de sol $\mathrm{j}$. 


\section{RESULTATS}

Cultures pratiquées : Le mil [Pennisetum glaucum (L.)R

l'arachide (Arachis hypogaea Linn.), le voandzou [Vigna $\mathrm{Br}$.] constitue la culture principale pratiquée par les populations des Communes de Simiri et de Tamou (Figure 2). L'oseille (Hibiscus sabdariffa L.), le sésame (Sesamum alatum Thonning), le maïs (Zea mays Linn.), subterranea (L.) Verdc.], le gombo (Hibiscus esculentus Linn.) et le fonio [Digitaria exilis (Kippist) Stapf] sont cultivés à faible proportion.

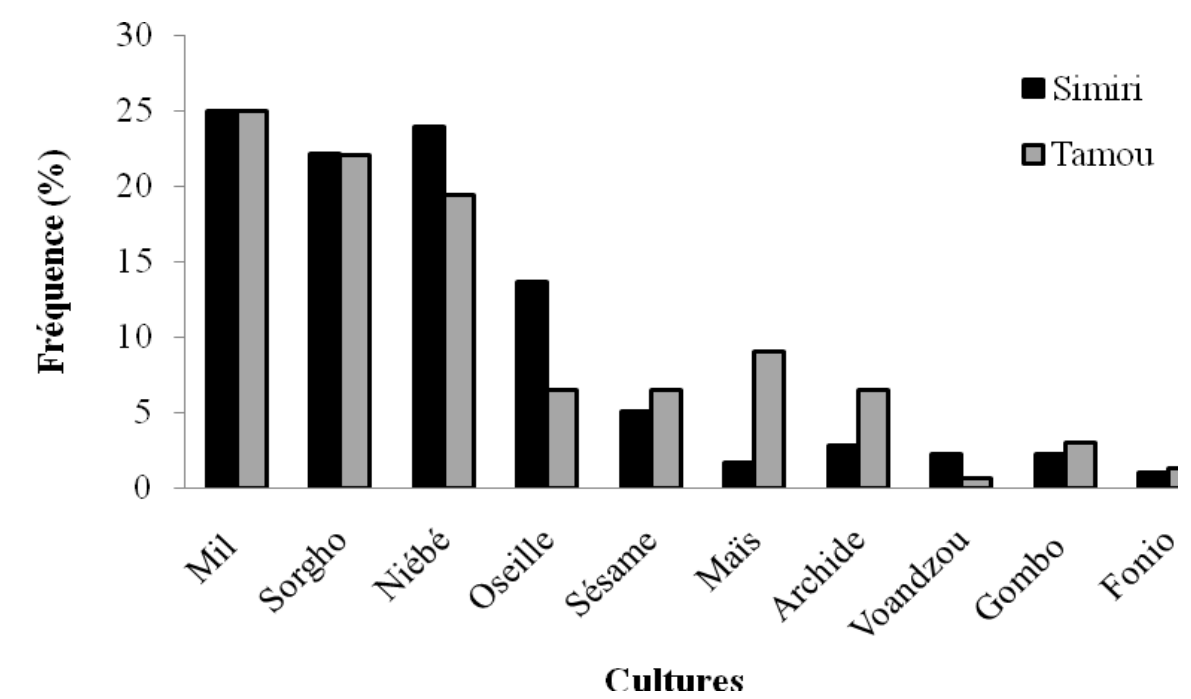

Figure 2 : Cultures pratiquées dans les terroirs de Simiri et de Tamou

Associations de cultures : La principale association de culture dans les deux Communes est l'association milniébé (Figure 3). Cependant, les associations de culture de mil-oseille, de mil-sésame, de sorgho-niébé-sésame et de mil-niébé-sésame sont les moins pratiquées.

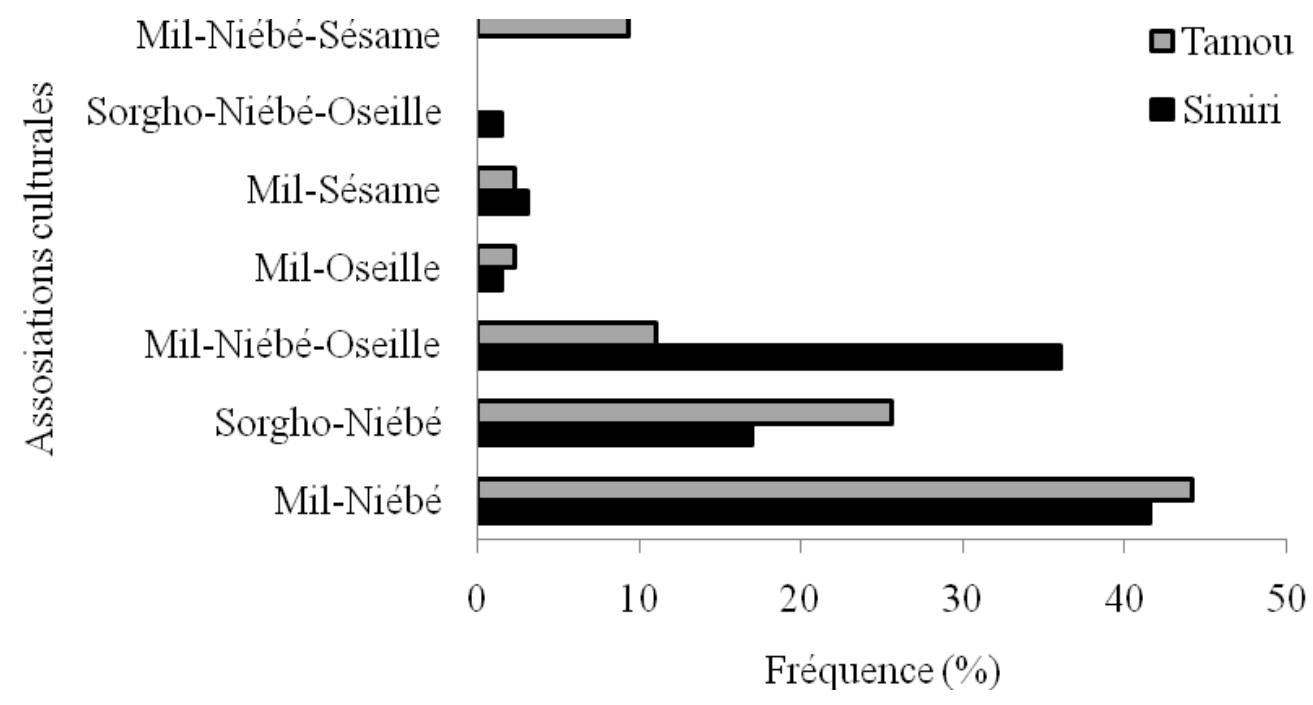

Figure 3 : Associations culturales dans les terroirs de Simiri et Tamou 
Bio-indicateurs paysans : Le plan factoriel de l'Analyse Factoriel de Correspondance (AFC) fait ressortir les bioindicateurs des types de sols pour chaque Commune (Figure 4). Les deux premiers axes (Dim 1 et 2), les plus significatifs de l'AFC expliquent $80,9 \%$ de la variance totale. Le tableau 3 montre que la fertilité des sols est perçue différemment dans les deux zones. Ainsi, les sols fertiles ont une productivité supérieure à 400 et à 800 $\mathrm{kg} / \mathrm{ha}$ en grains de mil respectivement à Simiri et à Tamou. Cependant, les mêmes mesures (400-800 kg/ha) sont enregistrées pour les sols fertiles de Simiri et les sols dégradés de Tamou. Par ailleurs, les espèces herbacées et les états de surface du sol caractérisent aussi chaque type de sol. C'est ainsi qu'à Tamou, les sols fertiles s'identifient à travers la nature infiltrant de la surface du sol et l'abondance de Pennisetum pedicellatum et Diheteropogon hagerupii. Quant aux sols dégradés de Simiri, eux sont colonisés par Mitracarpus scaber et des états de surface du sol de type ruisselant. Cependant, les sols fertiles à Simiri partagent les mêmes cortèges floristiques (Cenchrus biflorus et Sida cordifolia) que les sols dégradés à Tamou.

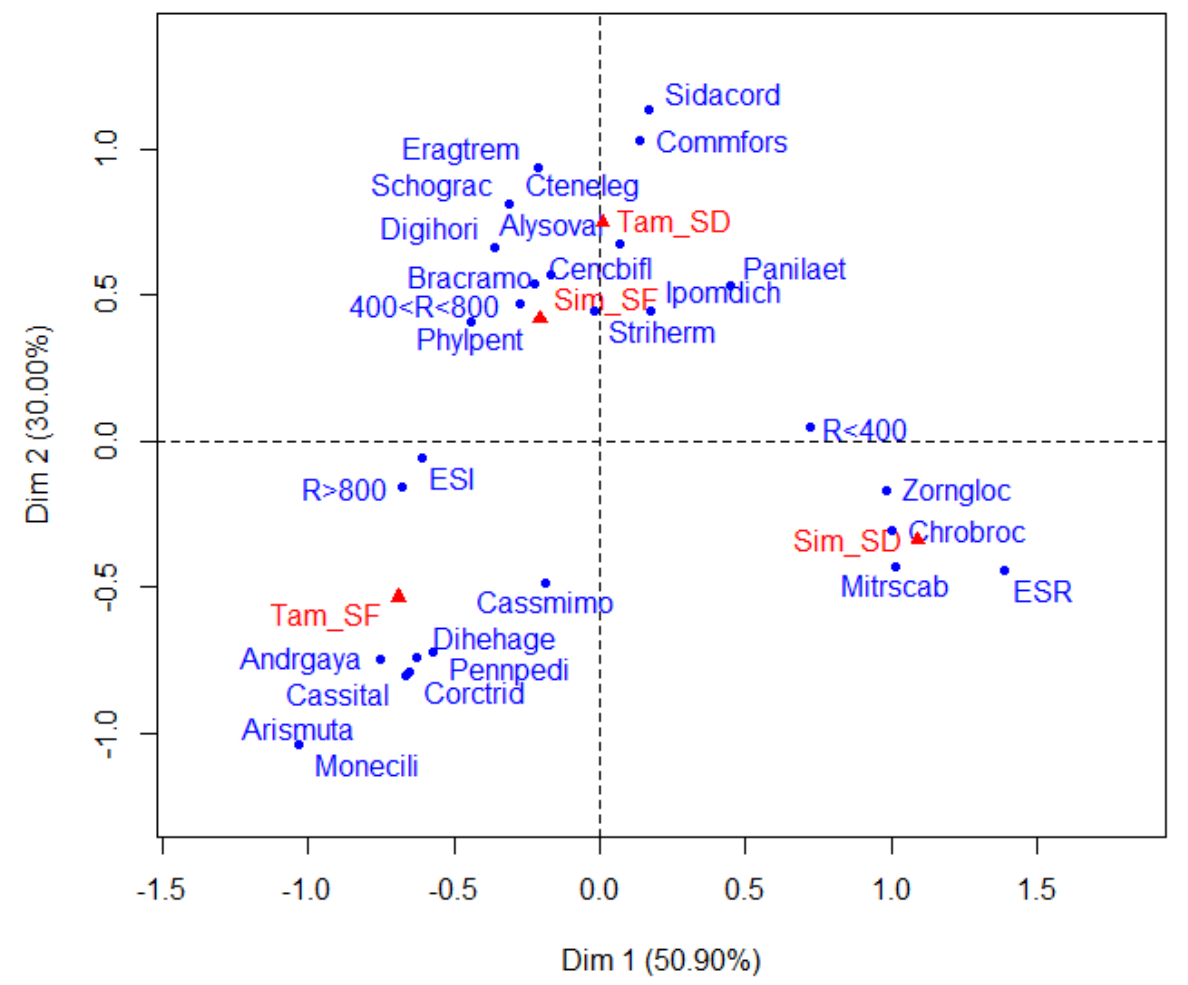

Figure 4 : Plan factoriel de l'Analyse Factoriel de Correspondance (AFC)

Sim_SD : Sols dégradés de Simiri ; Sim_SF : Sols fertiles de Simiri ; Tam_SD : Sols fertiles de Tamou ; Tam_SF : Sols fertiles de Tamou, ESR : état de surface ruisselant ; ESI : état de surface infiltrant; R : rendement en grains de mil ; Alisoval : Alysicarpus ovalifolius (Schum. Et Thonn.) J. Léonard., Andrgaya : Andropogon gayanus Kunth., Arismuta : Aristida mutabilis Trin. et Rupr., Bracramo : Brachiaria ramosa (L.) Stapf., Cassital : Cassia italica L., Cassmimo : Cassia mimosoides L., Cencbifl : Cenchrus biflorus Roxb., Chrobroc : Chrozophora brocchiana Vis., Commfors : Commelina forskoalei Vahl., Corctrid : Corchorus tridens L., Cteneleg : Ctenium elegans Kunth., Digihori : Digitaria horizontalis Willd., Dihehage : Diheteropogon hagerupii Hitchc., Eratrem : Eragrostis tremula Hochst. ex Steud., Ipomdich : Ipomoea dichroa Hochst. ex Choisy., Mitrscab : Mitracarpus scaber Zucc., Monecili: Monechma ciliatum (Jacq.) Milne. Red., Panilaet : Panicum laetum Kunth., Pennpedi : Pennisetum pedicellatum Trin., Phylpent : Phyllanthus pentandrus Schum. \& Thonn., Schograc : Schoenefeldia gracilis Kunth., Sidacord : Sida cordifolia L., Striherm : Striga hermonthica ; Zorngloc : Zornia glochidiata Reichb. Ex DC. 


\section{Moussa et al. . J. Appl. Biosci. Bio-indicateurs de la fertilité des sols et la perception paysanne à l'Ouest du Niger}

Tableau 3 : Valeurs des contributions et des cosinus carré des types de sols et des indicateurs paysans pour les deux premiers axes ( $\operatorname{Dim} 1$ et 2 ). Les variables et les facteurs ayant des valeurs en gras sont retenus pour l'interprétation avec les axes canoniques.

\begin{tabular}{lcccc}
\hline \multirow{2}{*}{ Type de sol et indicateurs paysans } & \multicolumn{3}{c}{ Dim 1 } & \multicolumn{2}{c}{ Dim 2 } \\
\cline { 2 - 5 } \multicolumn{1}{c}{ Sol fertile de Simiri } & 2,6 & 0,08 & $\mathbf{2 5 , 1}$ & $\mathbf{0 , 3 2}$ \\
Sol dégradé de Simiri & $\mathbf{6 4 , 8}$ & $\mathbf{0 , 9 1}$ & 3,7 & 0,09 \\
Sol fertile de Tamou & $\mathbf{3 2 , 5}$ & $\mathbf{0 , 6 1}$ & 33,2 & $\mathbf{0 , 3 7}$ \\
Sol dégradé de Tamou & 0,0 & 0,00 & $\mathbf{3 7 , 9}$ & $\mathbf{0 , 5 7}$ \\
Indicateurs paysans & & & & \\
Mitracarpus scaber Zucc. & $\mathbf{2 0 , 9}$ & $\mathbf{0 , 8 4}$ & 6,3 & 0,15 \\
Pennisetum pedicellatum Trin. & 3,7 & 0,32 & 10,2 & $\mathbf{0 , 5 1}$ \\
Diheteropogon hagerupii Hitchc. & 3,5 & 0,37 & $\mathbf{8 , 4}$ & $\mathbf{0 , 5 1}$ \\
Cenchrus biflorus Roxb., & 0,6 & 0,06 & $\mathbf{6 , 6}$ & $\mathbf{0 , 3 3}$ \\
Sida cordifolia L. & 0,1 & 0,01 & $\mathbf{9 , 3}$ & $\mathbf{0 , 5 4}$ \\
État de surface ruisselant & $\mathbf{1 7 , 9}$ & $\mathbf{0 , 8 9}$ & 3,1 & 0,09 \\
État de surface infiltrant & $\mathbf{6 , 8}$ & $\mathbf{0 , 7 2}$ & 0,1 & 0,01 \\
Rendement $<400$ & $\mathbf{1 7 , 8}$ & $\mathbf{0 , 9 9}$ & 0,1 & 0,01 \\
400< Rendement $<800$ & 1,9 & 0,23 & $\mathbf{9 , 8}$ & $\mathbf{0 , 6 8}$ \\
Rendement $>800$ & $\mathbf{1 1 , 5}$ & $\mathbf{0 , 9 0}$ & 1,0 & 0,05 \\
\hline
\end{tabular}

Valeurs indicatrices des espèces: Les valeurs indicatrices des espèces diffèrent selon la zone et le type de sol considérés (Tableau 4). C'est ainsi que pour les sols fertiles, les espèces qui enregistrent les valeurs indicatrices les plus élevés sont: Cassia mimosoides, Cenchrus biflorus et alysicarpus ovalifolius à Simiri contre Pennisetum pedicellatum, Alysicarpus ovalifolius et Corchorus tridens à Tamou. Par ailleurs, pour les sols dégradés, les espèces les plus fréquentes et les plus abondantes sont : Chrozophora brocchiana, Fimbristylis hispidula et Mitracarpus scaber à Simiri et Schizachyrium exile, Setaria pallide-fusca et Striga hermonthica à Tamou.

Tableau 4 : Valeur indicatrice des espèces selon le type de sol par Commune

\begin{tabular}{|c|c|c|c|c|}
\hline \multirow{2}{*}{ Espèces } & \multicolumn{2}{|c|}{ Sol fertile } & \multicolumn{2}{|c|}{ Sol dégradé } \\
\hline & Simiri & Tamou & Simiri & Tamou \\
\hline Acanthospermum hispidum DC. & 45 & 66 & 43 & 18 \\
\hline Alysicarpus ovalifolius (Schum. Et Thonn.) J. Léonard. & $78^{*}$ & $71^{*}$ & 32 & 49 \\
\hline Brachiaria ramosa (L.) Stapf. & 15 & 17 & 14 & 50 \\
\hline Cassia mimosoides $\mathrm{L}$. & $91^{*}$ & 64 & 57 & 60 \\
\hline Cassia obtusifolia L. & 60 & 56 & 57 & 58 \\
\hline Cenchrus biflorus Roxb. & $82^{*}$ & 41 & 19 & 35 \\
\hline Chrozophora brocchiana Vis. & 52 & 58 & $89^{*}$ & 50 \\
\hline Corchorus tridens $\mathrm{L}$. & 47 & $67^{*}$ & 42 & 25 \\
\hline Crotalaria macrocalyx Benth. & 9 & 23 & 56 & 20 \\
\hline Digitaria horizontalis Willd. & 28 & 53 & 43 & 18 \\
\hline Echinochloa colona (L.) Link. & 30 & 50 & 29 & 15 \\
\hline Fimbristylis hispidula (Vahl.) Kunth. & 22 & 44 & $81^{*}$ & 5 \\
\hline Kohautia virgata (Willd.) Bremeck. & 31 & 53 & 62 & 5 \\
\hline Merremia tridentata (L.) Hallier. f. & 10 & 12 & 40 & 20 \\
\hline Merremia pinnata (Choisy.) f. & 9 & 24 & 56 & 54 \\
\hline Mitracarpus scaber Zucc. & 76 & 5 & $80^{*}$ & 15 \\
\hline Panicum laetum Kunth. & 23 & 29 & 52 & 32 \\
\hline
\end{tabular}




\section{Moussa et al. . J. Appl. Biosci. Bio-indicateurs de la fertilité des sols et la perception paysanne à l'Ouest du Niger}

Pennisetum pedicellatum Trin.

Phyllanthus pentandrus Schum. \& Thonn.

Polycarpaea eriantha Hochst. ex A. Rich.

Schizachyrium exile (Hochst.) Pilger.

Sesamum alatum Thon.

Setaria pallide-fusca (Schumach.) Stapf \& C. E. Hubbard

Sida cordifolia L.

Striga hermonthica (Del.) Benth.

Tephrosia linearis (Willd.) Pers.

$\begin{array}{llll}46 & 74^{*} & 52 & 8 \\ 51 & 47 & 65 & 40 \\ 50 & 17 & 14 & 15 \\ 36 & 28 & 33 & 72^{*} \\ 20 & 23 & 77 & 20 \\ 55 & 46 & 52 & 61^{*} \\ 30 & 12 & 28 & 58 \\ 34 & 20 & 43 & 61^{*} \\ 11 & 29 & 75 & 25\end{array}$

* Valeur retenue

\section{DISCUSSION}

En zone sahélienne, les faibles des rendements de cultures constituent une des conséquences majeures de la baisse de la fertilité des sols (Pieri, 1992). Des enquêtes réalisées auprès des populations des Communes de Simiri et de Tamou à l'Ouest du Niger montrent que le mil est majoritairement cultivé en association avec le niébé ou le sorgho. Selon la direction des statistiques du Ministère de l'agriculture (MA/DS., 2013), le mil est la principale culture à l'Ouest du Niger. Cela pourrait s'expliquer par sa forte capacité d'adaptation de cette culture aux sols sableux acide et pauvre en matière organique. Selon la perception paysanne, un sol est considéré fertile si le rendement en grains de mil est supérieur à $800 \mathrm{~kg} / \mathrm{ha}$ à Tamou et à 400 $\mathrm{kg} / \mathrm{ha}$ à Simiri. En effet, le rendement moyen en grains de mil de 2000 à 2013 est de 561 kg/ha à Say (Tamou) et $333 \mathrm{~kg} / \mathrm{ha}$ à Ouallam (Simiri) (MA/DS., 2013). Ainsi, sur la base des seuils de rendement donnés par les paysans, la plupart des sols sont dégradés dans les deux Communes. Toutefois une tendance est observée au profit des sols de Tamou. Ces différences de rendements peuvent s'expliquer d'une part par les conditions pluviométriques et d'autre part, par l'état de fertilité chimique des sols. En effet, les moyennes pluviométriques (1981-2002) enregistrées ont une tendance plus élevée à Tamou $(601,7 \mathrm{~mm})$ qu'à Simiri (330,8 mm) (Mahamane et al., 2013). La fertilité du sol à travers les teneurs en carbone organique et la capacité d'échange cationique sont significativement élevés à Tamou $\left(1,9 \pm 0,3 \mathrm{~g} \mathrm{~kg}^{-1} ; 2,9 \pm 0,6 \mathrm{cmol}_{\mathrm{c}} \mathrm{kg}^{-1}\right)$ qu'a Simiri $\left(1,2 \pm 0,6 \mathrm{~g} \mathrm{~kg}^{-1} ; 1,6 \pm 0,5 \mathrm{cmol}_{\mathrm{c}} \mathrm{kg}^{-1}\right)$ (Moussa et al., 2015). De plus, pour différencier ces sols, les paysans utilisent les mauvaises herbes et les états de surface du sol. C'est ainsi que la présence dans les champs de $C$. biflorus (Simiri), $P$. pedicellatum et $D$. hagerupii (Tamou) est synonyme de la fertilité des sols tandis que $M$. scaber
(Simiri) et S. cordifolia (Tamou) sont considérés comme espèces indicatrices de la dégradation des sols. Les travaux de Soumana (1992) réalisés dans la même zone d'étude ont montrés que Andropogon gayanus, Aristida mutabilis, Chloris pilosa colonisaient les sols fertiles tandis que Eragrostis tremula et $C$. biflorus se développaient sur les sols dégradés. Cependant, la comparaison de ces deux listes d'espèces indicatrices fait ressortir un changement d'état de ces agrosystèmes dans le temps et dans l'espace. En effet, la présence d'une espèce en un lieu donné est une valeur informative qui est différente d'une espèce à l'autre pour un même cortège floristique (Balent \& Fily, 1991). Ce changement d'état se traduit par une perception différente de la valeur indicatrice de l'espèce à une échelle spatio-temporelle. C'est ainsi que $C$. biflorus qui était reconnue par les populations comme une espèce indicatrice des sols dégradés (dans les années 90) est considérée maintenant comme indicatrice des sols fertiles. De plus, les relevés de végétation effectués montrent que ces espèces ont des valeurs indicatrices élevés. Cela montre qu'au-delà de la présence de l'espèce, sa fréquence et son abondance sont des valeurs informatives considérées par les paysans dans l'identification des espèces indicatrices. Par ailleurs, au Cameroun, les espèces les plus abondantes ont été considérées comme « espèces guides » lors de la détermination du niveau de fertilité d'un sol (M'Biandoun et al., 2006). Les états de surface du sol sont de nature infiltrant pour les sols fertiles et de type ruisselant pour les sols dégradés. En effet, une augmentation du stock hydrique du sol à travers l'infiltration des eaux de pluies favorise la croissance des cultures et améliore le rendement. Selon Somé et al (1999), la fertilité d'un sol apparait comme une réalité physique traduite par la présence d'une espèce végétale ou par l'aspect du sol. 


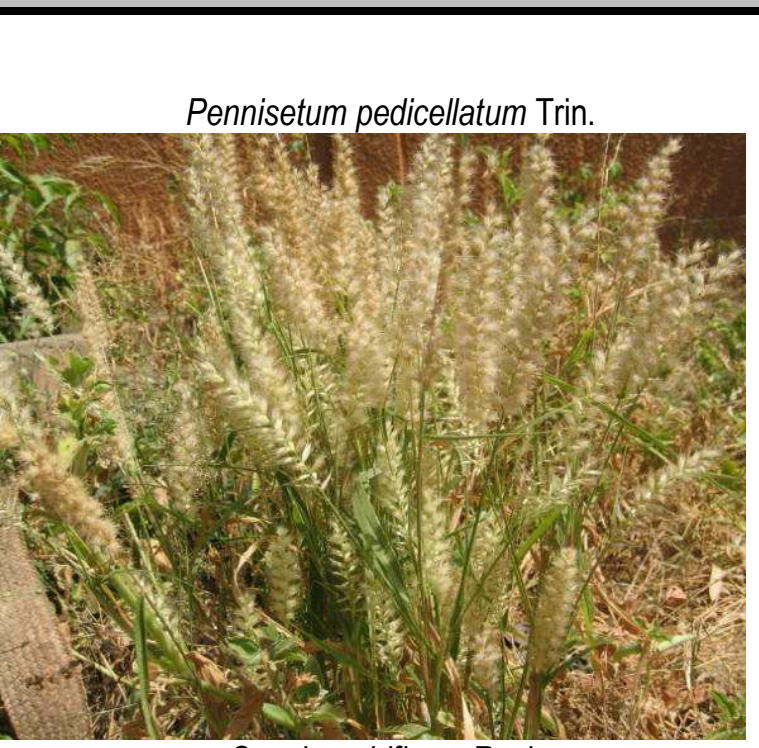

Cenchrus biflorus Roxb.

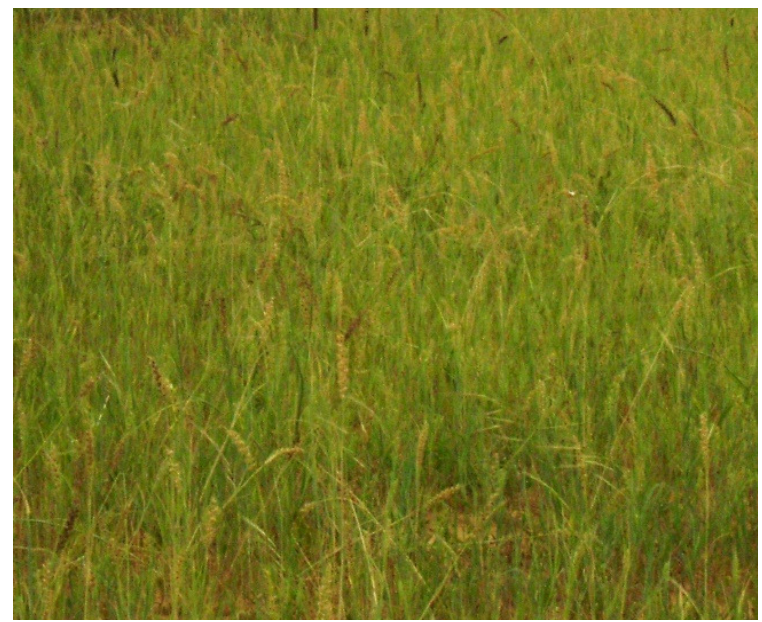

Photos 1 : quelques espèces indicatrices

\section{CONCLUSION}

Les résultats de cette étude ont montrés que les populations des Communes de Simiri et de Tamou cultivent majoritairement le mil en association avec le niébé ou le sorgho. Ainsi, des rendements en grains de mil supérieurs à $800 \mathrm{~kg} / \mathrm{ha}$ (Tamou) et à $400 \mathrm{~kg} / \mathrm{ha}$ (Simiri) amènent les paysans à qualifier un sol comme

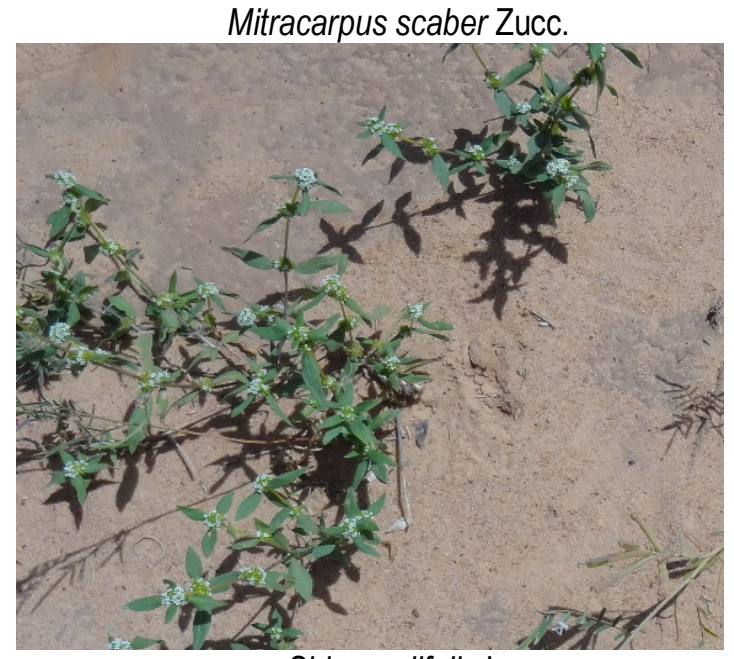

Sida cordifolia L.

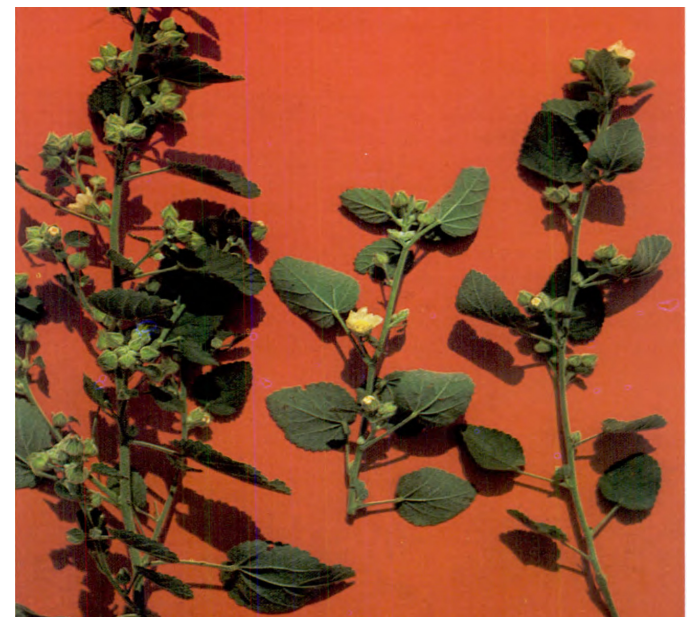

fertile. De plus, $C$. biflorus (Simiri), P. pedicellatum et $D$. hagerupii (Tamou) sur des états de surface du sol de nature infiltrant sont considérés comme indicateurs de la fertilité des sols tandis que M. scaber (Simiri) et $S$. cordifolia (Tamou) sur des sols ruisselants est synonyme de la dégradation des sols.

\section{REMERCIEMENT}

Nous remercions toutes les personnes ressources qui ont contribuées à l'élaboration de ce document.

\section{REFERENCES}

Ambouta J.M.K., 1997. Définition et caractérisation des structures de végétation contractée au sahel: cas de la brousse tigrée de l'Ouest Nigérien. In : d'Herbès J.M., Ambouta J.M.K., Peltier R., éds. Fonctionnement et gestion des écosystèmes forestiers contractés sahéliens. John Libbey Eurotext, Paris, 41-57.

Balent G. et Fily M., 1991. Dynamique de la végétation selon les pratiques des agriculteurs: une modélisation à partir des données recueillies 
dans la vallée des Pyrénées centrales, Études et recherches sur les systèmes de production et le développement, $\mathrm{N}^{\circ} 23$, Paris, Inra, $48 \mathrm{p}$.

Bationo A. et Buerkert A., 2001. Soil Organic carbon measurement for sustainable land use in Sudano-Sahelian West Africa. Nutr. Cycl. Agroecosys., 61, 131-142.

Braun-Blanquet J., 1932. Plant sociology the study of plant communities. Ed. Mc Gray Hill, New York, London, 439p.

Casenave A. et Valentin C., 1989. Les états de surface de la zone sahélienne : influence sur l'infiltration. Edition de ORSTOM,Paris, $229 \mathrm{p}$.

Courault D., D'Herbes J.M. et Valentin C., 1990. Le bassin versant de Sama Dey: Premières observation pédologiques et phytoécologiques. ORSTOM, Paris, 31p.

Diouf A., Barbier N., Mahamane A., Lejoly J., Saadou M. et Bogaert J. 2010. Caractérisation de la structure spatiale des individus ligneux dans une "Brousse tachetée" au Sud-ouest du Niger. Canadian Journal of Forest Research, 40, 827835.

Dufrene M. et Legendre P., 1997. Species Assemblages and Indicator Species: The Need for a Flexible Asymmetrical Approch. Ecol. Monogr., 67, 3, 345-366.

FAO., 2003. Gestion de la fertilité des sols pour la sécurité alimentaire en Afrique subsaharienne. Rome. Italie, $63 \mathrm{p}$.

MA/DS, 2013. Évaluation des récoltes de la Campagne agricole d'hivernage 2012 et résultats définitifs 2012-2013. République du Niger, Ministère de l'Agriculture, Direction des Statistiques, Rapport National de Synthèse, 39p.

Loireau M., 1998. Espace, Ressources, Usages : Interactions milieux et sociétés dans le Sahel nigérien. Thèse Doctorat, géographie, Université Paul Valery, Montpellier 3, $411 \mathrm{p}$.

Mahamane A., Morou B., Zaman-Allah M., Saadou M., Saley K., Bakasso Y... Jauffret S., 2013. Climate Variability in Niger : Potential Impacts on Vegetation Distribution and Productivity. Journal of Environmental Science and Engineering B.1.49-57

M'Biandoun M., Guibert H. et Olina J.P., 2006. Caractérisation de la fertilité du sol en fonction des mauvaises herbes présentes. Tropicultura, 24, 4, 247-252.
M'Biandoun M. et Bassala J-P. O., 2007. Savoir paysan et fertilité des terres au Nord-Cameroun. Cahiers Agricultures 16, 3 .

Moussa M. Boubacar, Diouf A., Abdourahamane S.I., Jangorzo N. S., Mahamane A., Axelsen J.A., Ambouta J.M.K., 2015. Soil Physicochemical Characteristics Under Different Ecosystems in Western Niger. Int.J.Curr.Microbiol.App.Sci 4,4, 375-383.

Pieri C. 1992. Fertility of Soils: A Future for farming in the West African Savannah. Springer, Berlin, 348pp.

Pontanier R., Mhir I.A., Akrlml N., Aronson J. \& LE Floc'h E., 1995 : L'homme peut-il refaire ce qu'il a défait ? (edition) John Libbey Eurotext, Paris. $455 \mathrm{p}$.

$\mathrm{R}$ Development Core Team, 2010. A language and environment for statistical computing. $R$ Foundation for Statistical Computing, Vienna, Austria. ISBN 3-900051-07-0, URL: http://www.R-project.org.

Roger P. \& Christian F., 2002. Les indicateurs du fonctionnement et du changement du milieu rural. Jean-Yves Jamin, L. Seiny Boukar, Christian Floret. Cirad - Prasac, $10 \mathrm{p}$.

Saadou M., 1990. La végétation des milieux drainés nigériens à l'est du fleuve Niger. Thèse de doctorat, Université Niamey, Niger, $393 p$.

Somé A, Alexandre D-Y et Hien V., 1999. Bio-indicateurs paysans de la fertilité des sols et gestion du cycle culture-jachère en zone soudanienne (Burkina-Faso) in JATBA (Journal d'Agriculture Traditionelle et de Botanique Appliquée), 41 (2) p. 137-146.

Soumana I, 1992. Comment les paysans différencient-ils les sols fertiles des sols pauvres au Niger? Séminaire sur les recherches au sylvopastoralisme au Sahel, 7-12 mai 1992. Dakar : Projet RCS Sahel, UNESCO-MAB. 